\title{
Combinatorial Bounds for Authentication Codes with Arbitration
}

\author{
Kaoru KUROSAWA and Satoshi OBANA \\ Department of Electrical and Electronic Engineering, \\ Faculty of Engineering, Tokyo Institute of Technology \\ 2-12-1 O-okayama, Meguro-ku, Tokyo 152, Japan \\ E-mail kkurosaw@ss.titech.ac.jp
}

\begin{abstract}
Unconditionally secure authentication codes with arbitration ( $A^{2}$-codes) protect against deceptions from the transmitter and the receiver as well as that from the opponent.

In this paper, we present combinatorial lower bounds on the cheating probabilities for $A^{2}$-codes in terms of the number of source states, that of the whole messages and that of messages which the receiver accepts as authentic for each source state. Previously, only entropy based lower bounds were known. Our bounds for the model without secrecy are tight because the $A^{2}$-codes given by Johansson meet our bounds with equality.
\end{abstract}

\section{Introduction}

In the model of unconditionally secure authentication codes ( $A$-codes) [1], there are three participants, a transmitter, a receiver and an opponent. The opponent tries to cheat the receiver by impersonation attack and substitution attack. This model has been studied extensively so far. Lower bounds on the cheating probabilities based on entropy were given by $[2,3]$. Combinatorial lower bounds were given by $[4,5,6,7,8,9]$. In this model, the transmitter and the receiver are both honest and trust each other. However, it is not always the case that the two parties want to trust each other.

Inspired by this problem, Simmons introduced an extended model, $A^{2}$-code model, in which there is a fourth person, an arbiter [10,11]. In this model, caution is taken against deception of the transmitter and the receiver as well as that of the opponent. The arbiter has access to all key information of the transmitter and the receiver, and solves disputes between them. We denote by $E_{R}$ the set of keys of the receiver and by $E_{T}$ denotes the set of keys of the transmitter, respectively.

In this model, there are essentially five different kinds of cheatings, impersonation by the opponent, substitution by the opponent, impersonation by the transmitter, impersonation by the receiver and substitution by the receiver. Denote these cheating probabilities by $P_{I}, P_{S}, P_{T}, P_{R_{0}}$ and $P_{R_{1}}$. Johansson showed an entropy based lower bound on these five cheating probabilities [12]. By assuming $\max \left(P_{I}, P_{S}, P_{T}, P_{R_{0}}, P_{R_{1}}\right)=1 / q$, he also showed a lower bound on the size of keys in terms of $q[12]$. Recently, Kurosawa showed a more tight lower bound 
for a larger set of source states by assuming $P_{I}=P_{S}=P_{T}=P_{R_{0}}=P_{R_{1}}=1 / q$ [15]. However, combinatorial lower bounds on the cheating probabilities are not known. (The structure of $A^{2}$-codes is not well known.)

In this paper, we present combinatorial lower bounds on $P_{I}, P_{S}, P_{T}, P_{R_{0}}, P_{R_{1}}$, $\left|E_{R}\right|$ and $\left|E_{T}\right|$. First, we show the following bounds for a $A^{2}$-code model without secrecy. Let $|S|$ denote the number of source states and $|M|$ denote that of messages, respectively. Assume that each $f \in E_{R}$ accepts $c$ messages for each source state $s$. Let $l \triangleq|M| / c|S|$. Then $P_{I} \geq 1 / l$ and $P_{T} \geq(c-1)|S| /(|M|-|S|)$. If $P_{I}=1 / l$, then $P_{S} \geq 1 / l$. For a separable case, if $P_{I}=1 / l$, then $P_{T} \geq 1 / l$. If $P_{I}=P_{S}=P_{T}=1 / l$, then $\left|E_{R}\right| \geq c|S|(l-1)+1$. Similar bounds are obtained for $P_{R_{0}}, P_{R_{1}}$ and $\left|E_{T}\right|$. Our bounds on $P_{I}, P_{S}, P_{T}, P_{R_{0}}$ and $P_{R_{1}}$ are tight because the $A^{2}$-codes given by Johansson $[13,14]$ (in which $c=l=q$ ) meet our bounds with equality.

Further, we show such combinatorial lower bounds for general $A^{2}$-codes.

\section{Preliminaries}

\subsection{Authentication code ( $A$-code)}

In the model of $A$-codes, there are three participants, a transmitter $\mathrm{T}$, a receiver $\mathrm{R}$ and an opponent $\mathrm{O}$. The transmitter $\mathrm{T}$ and the receiver $\mathrm{R}$ share a common encoding rule $e$. On input a source state $s, \mathrm{~T}$ computes a message $m=e(s)$ and sends $m$ to $\mathrm{R}$. $\mathrm{R}$ accepts or rejects $m$ based on $e$. An $A$-code is called an $A$-code without secrecy if a source state is uniquely determined from a message $m$. It is possible that more than one message can be used to communicate a particular source state; this is called splitting. Defining

$$
M(e, s) \triangleq\{m \mid e(s)=m\}
$$

splitting means $|M(e, s)|>1$. If $|M(e, s)|=1$ for $\forall e$ and $\forall s$, the $A$-code is called an $A$-code without splitting.

We assume independent probability distributions on source states and on encoding rules, respectively. In the impersonation attack, the opponent $O$ sends a message $m$ to the receiver. $O$ succeeds if $m$ is accepted by the receiver as authentic. The impersonation attack probability $P_{I}$ is defined by

$$
P_{I} \triangleq \max _{m \in M} \operatorname{Pr}[\mathrm{R} \text { accepts } m]
$$

In the substitution attack, $\mathrm{O}$ observes a message $m$ that is transmitted by $\mathrm{T}$ and substitutes $m$ with another message $\hat{m}$. O succeeds if $\hat{m}$ is accepted by the receiver as authentic. For no splitting, the substitution attack probability $P_{S}$ is defined by

$$
P_{S} \triangleq \sum_{m \in M} \operatorname{Pr}(M=m) \max _{\hat{m} \neq m} \operatorname{Pr}[\mathrm{R} \text { accepts } \hat{m} \mid \mathrm{R} \text { accepts } m]
$$


For splitting, the maximum is taken over $\hat{m}$ such that the source state conveyed by $\hat{m}$ is different from that of $m$. Let $S \triangleq\{s\}, E \triangleq\{e\}$ and $M \triangleq\{m\}$. We denote an $A$-code by $(S, E, M)$.

Proposition 1. [4] In an A-code without splitting, $P_{I} \geq|S| /|M|$. The equality holds if and only if $\operatorname{Pr}[\mathrm{R}$ accepts $m]=|S| /|M|$ for $\forall m$.

Proposition 2. [7] In an A-code without splitting and without secrecy, if $P_{I}=$ $|S| /|M|$, then $P_{S} \geq|S| /|M|$.

Definition 3. An orthogonal array $O A(l, k, \lambda)$ is a $\lambda l^{2} \times k$ array of $l$ symbols such that, in any two columns of the array, every one of the possible $l^{2}$ pairs of symbols occurs in exactly $\lambda$ rows.

Proposition 4. Suppose we have an A-code without splitting and without secrecy such that $P_{I}=P_{S}=|S| /|M|=1 / l$.

1. $|E| \geq|S|(l-1)+1$. The equality occurs if and only if the incidence matrix of $E$ is an orthogonal array $O A(l,|S|, \lambda)$, where $\lambda=(|S|(l-1)+1) / l^{2}$ and each $e \in E$ is used with equal probability [8].

2. Also, $|E| \geq l^{2}[7]$.

Proposition 5. [9] In a splitting $A$-code, let $M(e) \triangleq\{m \mid$ e accepts $m\}$. Then,

$$
P_{I} \geq \min _{e \in E} \frac{|M(e)|}{|M|} \quad P_{S} \geq \min _{e \in E} \frac{|M(e)|-\max _{s \in S}|M(e, s)|}{|M|-\min _{s \in S}|M(e, s)|}
$$

\subsection{Authentication code with arbitration $\left(A^{2}\right.$-code)}

We denote an $A^{2}$-code by $\left(S, M, E_{R}, E_{T}\right)$, where $S=\{s\}$ is a set of source states, $M=\{m\}$ is a set of messages, $E_{R}=\{f\}$ is a set of the receiver's decoding rules and $E_{T}=\{e\}$ is a set of the transmitter's encoding rules.

The selection of $e$ and $f$ may be done in several ways. One choice is to let the receiver $\mathrm{R}$ choose his $f$ and then secretly pass this on to the arbiter. In this case, the arbiter constructs $e$ and passes this on to the transmitter $\mathrm{T}$. Another choice is to do the other way around and the third approach is to let the arbiter construct both rules. In any case, on input $s$, T sends $m$ such that $m=e(s)$ to R. R accepts $m$ iff $f(m)$ is valid. The arbiter accepts $m$ as authentic iff $e$ can generate $m$.

In this model, there are five different kinds of attacks.

$I$, Impersonation by the opponent. The cheating probability $P_{I}$ is defined in the same way as eq.(2.1). $S$, Substitution by the opponent. The cheating probability $P_{S}$ is defined in the same way as eq.(2.2). $T$, Impersonation by the transmitter. The transmitter sends a message to the receiver and denies having sent it. The transmitter succeeds if the message is accepted by the receiver as authentic and if 
the message is not one of the messages that the transmitter could have generated due to his encoding rule. This cheating probability $P_{T}$ is defined as follows

$$
P_{T} \triangleq \max _{e \in E_{T}} \max _{m \in M} \operatorname{Pr}[\mathrm{R} \text { accepts } m \text { and } m \text { is not generated by } e \mid \mathrm{T} \text { has } e]
$$

$R_{0}$, Impersonation by the receiver. The receiver claims to have received a message from the transmitter. The receiver succeeds if the message could have been generated by the transmitter due to his encoding rule. This cheating probability $P_{R_{0}}$ is defined by

$$
P_{R_{0}} \triangleq \max _{f \in E_{R}} \max _{m \in M} \operatorname{Pr}\left[\text { Arbiter (or } \mathrm{T} \text { ) accepts } m \mid \mathrm{R} \text { has } f \in E_{R}\right]
$$

$R_{1}$, Substitution by the receiver. The receiver receives a message from the transmitter but claims to have received another message. The receiver succeeds if this other message could have been generated by the transmitter due to his encoding rule. This cheating probability $P_{R_{1}}$ is defined by

$$
\begin{aligned}
P_{R_{1}} \triangleq & \max _{f \in E_{R}} \sum_{m \in M} \operatorname{Pr}(m) \\
& \max _{\hat{m} \neq m} \operatorname{Pr}[\text { Arbiter (or } \mathrm{T} \text { ) accepts } \hat{m} \mid \mathrm{R} \text { has } f \text { and } \mathrm{T} \text { sends } m]
\end{aligned}
$$

Let

$$
E_{R} \circ E_{T} \triangleq\left\{(e, f) \mid \operatorname{Pr}\left[\mathrm{T} \text { has } e \in E_{T} \text { and } \mathrm{R} \text { has } f \in E_{R}\right]>0\right\}
$$

For $A^{2}$-codes, Johansson showed an information theoretic bound such as follows.

Proposition 6. [12] In an $A^{2}$-code without splitting

$$
\begin{array}{ll}
P_{I} \geq 2^{-\inf I\left(E_{R} ; M\right)} & P_{R_{0}} \geq 2^{-\inf I\left(E_{T} ; M \mid E_{R}\right)} \\
P_{S} \geq 2^{-\inf I\left(E_{R} ; M^{\prime} \mid M\right)} & P_{R_{1}} \geq 2^{-\inf I\left(E_{T} ; M^{\prime} \mid E_{R}, M\right)} \\
P_{T} \geq 2^{-\inf I\left(E_{R} ; M \mid E_{T}\right)} &
\end{array}
$$

where $I(X ; Y)$ denotes the mutual entropy of $X$ and $Y$.

Proposition 7. [12]

$$
\begin{aligned}
& \left|E_{R}\right| \geq\left(P_{I} P_{S} P_{T}\right)^{-1} \\
& \left|E_{T}\right| \geq\left(P_{I} P_{S} P_{R_{0}} P_{R_{1}}\right)^{-1}
\end{aligned} \quad\left|E_{R} \circ E_{T}\right| \geq\left(P_{I} P_{S} P_{T} P_{R_{0}} P_{R_{1}}\right)^{-1}
$$

\section{Combinatorial bounds for $\boldsymbol{A}^{2}$-codes without secrecy}

In this section, we present combinatorial lower bounds for $A^{2}$-codes without splitting and without secrecy. To derive our bounds, we develop three techniques. The first technique is a reduction of an $A^{2}$-code to an $A$-code. The second one is a restriction of messages which is described by the following Theorem. 
Theorem 8. For an $A$-code $(S, E, M)$, consider a subcode $(S, E, \hat{M})$ such that $\hat{M} \subseteq M$. Then, for $\forall m \in \hat{M}$,

$$
\begin{aligned}
& \operatorname{Pr}[R \text { accepts } m \text { in the original } A \text {-code }] \\
= & \operatorname{Pr}[R \text { accepts } m \text { in the subcode }]
\end{aligned}
$$

The third technique is given by the following Theorem. This technique will be a basic Theorem for $A$-codes.

Theorem 9. In an $A$-code without splitting $(S, E, M)$, if $\operatorname{Pr}[R$ accepts $m]=1 / l$ for $\forall m$, then $|M|=|S| l$.

Proof. Let $X=\left\{x_{i j}\right\}$ be the incidence matrix of $E$. That is,

$$
x_{i j}= \begin{cases}1 & \text { if } e_{i} \in E \text { accepts } s_{j} \in S \\ 0 & \text { otherwise }\end{cases}
$$

Then, $\operatorname{Pr}\left[\mathrm{R}\right.$ accepts $\left.m_{j}\right]=\sum_{i} \operatorname{Pr}\left[e=e_{i}\right] x_{i j}$. If $\operatorname{Pr}\left[\mathrm{R}\right.$ accepts $\left.m_{j}\right]=1 / l$ for $\forall m_{j}$, then

$$
\begin{aligned}
|M| / l & =\sum_{j} \operatorname{Pr}\left[\mathrm{R} \text { accepts } m_{j}\right]=\sum_{j} \sum_{i} \operatorname{Pr}\left[e=e_{i}\right] x_{i j} \\
& =\sum_{i} \operatorname{Pr}\left[e=e_{i}\right] \sum_{j} x_{i j}=\sum_{i} \operatorname{Pr}\left[e=e_{i}\right]|S| \\
& =|S|
\end{aligned}
$$

Hence, $|M|=l|S|$.

For an $A^{2}$-code $\left(S, M, E_{R}, E_{T}\right)$, we define a decoding rule of the receiver $f \in E_{R}$ as follows.

$$
f(m)= \begin{cases}s \in S & \text { if } m \text { is accepted as } s \\ \text { reject } & \text { if } m \text { is rejected. }\end{cases}
$$

Let

$$
\begin{aligned}
M(f, s) & \triangleq\{m \mid f(m)=s\} \\
M(f) & \triangleq\{m \mid f \text { accepts } m\}\left(=\bigcup_{s \in S} M(f, s)\right) \\
M_{s} & \triangleq\left\{m \mid f(m)=s, f \in E_{R}\right\}\left(=\bigcup_{f \in E_{R}} M(f, s)\right) \\
E_{R}(e) & \triangleq\{f \mid \operatorname{Pr}[\mathrm{R} \text { has } f \mid \mathrm{T} \text { has } e]>0\}
\end{aligned}
$$




\subsection{Lower bound on $P_{I}$ and $P_{S}$}

To prevent the impersonation attack of the receiver, it must be that $|M(f, s)|>$ 1. That is, from a view point of the receiver, $A^{2}$-codes must be splitting. To derive our bounds, we assume the following assumption.

Assumption 10. $|M(f, s)|=c$ for $\forall f \in E_{R}$ and $\forall s \in S$.

That is, each $f \in E_{R}$ accepts $c$ messages for each $s \in S$. For each source state $s_{i} \in S$, define

$$
S_{i} \triangleq\left\{s_{i 1}, s_{i 2}, \ldots, s_{i c}\right\}, \quad \hat{S} \triangleq S_{1} \cup S_{2} \cup \ldots
$$

Then, we can consider an $A$-code without splitting $\left(\hat{S}, E_{R}, M\right)$ which corresponds to the original $A^{2}$-code $\left(S, M, E_{R}, E_{T}\right)$ in a natural way. Clearly, $|\hat{S}|=c|S|$.

Theorem 11. Under assumption $10, P_{I} \geq c|S| /|M|$. The equality holds if and only if

$$
\operatorname{Pr}[R \text { accepts } m]=c|S| /|M| \text { for } \forall m \in M
$$

Proof. It is clear that $P_{I}$ of the $A^{2}$-code is equal to the impersonation attack probability for the splitting $A$-code $\left(\hat{S}, E_{R}, M\right)$. Apply proposition 1 to $\left(\hat{S}, E_{R}, M\right)$.

Assumption 12. $P_{I}=c|S| /|M|=1 / l$.

Theorem 13. Under assumption 10 and 12, $\left|M_{s}\right|=c l$ for $\forall s \in S$.

Proof. From assumption 12 and theorem 11,

$$
\operatorname{Pr}[\mathrm{R} \text { accepts } m]=1 / l \text { for } \forall m \in M
$$

We consider a subcode of $\left(\hat{S}, E_{R}, M\right)$ such that the set of messages is restricted to $M_{s}$. This is an $A$-code without splitting in which the number of messages is $\left|M_{s}\right|$ and that of source states is $c$ (from assumption 10). Even for this restricted $A$-code, we have

$$
\operatorname{Pr}[\mathrm{R} \text { accepts } m]=1 / l \text { for } \forall m \in M_{s}
$$

from Theorem 11. Then, from Theorem $9,\left|M_{s}\right|=c l$

Theorem 14. Under assumption 10 and 12,

$$
P_{S} \geq 1 / l
$$

Proof. $P_{S}$ is defined by

$$
P_{S} \triangleq \sum_{m^{\prime} \in M} \operatorname{Pr}\left[\mathrm{T} \text { sends } m^{\prime}\right] \max \operatorname{Pr}\left[\mathrm{R} \text { accepts } m \mid \mathrm{R} \text { accepts } m^{\prime}\right]
$$


Let $m^{\prime} \in M_{s_{i}}$. Then, the maximum is taken over $m \in M \backslash M_{s_{i}}$. For $m^{\prime} \in M_{s_{i}}$, define

$$
P_{I}^{\prime}\left(m^{\prime}\right) \triangleq \max _{m \in M \backslash M_{\mathbf{s}_{i}}} \operatorname{Pr}\left[\mathrm{R} \text { accepts } m \mid \mathrm{R} \text { accepts } m^{\prime}\right] .
$$

Then, this is the impersonation attack probability against an $A$-code without splitting $\left(\hat{S} \backslash S_{i}, E_{R}, M \backslash M_{s_{i}}\right.$ ), where the probability distribution on $E_{R}$ is conditioned by the fact that $\mathrm{R}$ accepts $m^{\prime}$. Then, from proposition 1 ,

$$
P_{I}^{\prime}\left(m^{\prime}\right) \geq \frac{\left|\hat{S} \backslash S_{i}\right|}{\mid M \backslash M_{s_{i} \mid}} .
$$

Now

$\left|\hat{S} \backslash S_{i}\right|=|\hat{S}|-\left|S_{i}\right|=c|S|-c=c(|S|-1), \quad\left|M \backslash M_{s_{i}}\right|=|M|-\left|M_{s_{i}}\right|=c l|S|-c l$

from assumption 12 and Theorem 13. Therefore, we have $P_{I}^{\prime}\left(m^{\prime}\right) \geq 1 / l$ for $\forall m^{\prime}$. Then, we have eq.(3.2) from eq.(3.3) and eq.(3.4).

\subsection{Lower bound on $\boldsymbol{P}_{T}$}

Let $h \triangleq \min _{e \in E_{T}}\left|E_{R}(e)\right|$.

Theorem 15. Under assumption 10, $P_{T} \geq \max \{(c-1)|S| /(|M|-|S|), 1 / h\}$.

Proof. It is easy to see that $P_{T} \geq 1 / h$.

Suppose that the transmitter $\mathrm{T}$ has an encoding rule $e \in E_{T}$. Let $m_{i}=e\left(s_{i}\right)$ for $i=1,2, \ldots,|S|$. T must send $m$ to $\mathrm{R}$ such that $m \neq m_{i}$ for $\forall i$ for cheating. Consider the following subcode of $\left(\hat{S}, E_{R}, M\right)$ such as follows. Let $X$ be the incidence matrix of $E_{R}(e)$ which is a $\left|E_{R}(e)\right| \times|M|$ binary matrix. Remove the columns corresponding to $\left\{m_{i}|i=1,2, \cdots| S \mid,\right\}$ from this matrix. Then, we obtain an incidence matrix of an $A$-code without splitting $\left(S^{\prime}, E_{R}(e), M \backslash\left\{m_{i}\right\}\right)$, where $\left|S^{\prime}\right|=|\hat{S}|-|S|=c|S|-|S|=(c-1)|S|$. Theorem 11 holds for this subcode. The best strategy of the transmitter is at least as good as the impersonation attack against this modified $A$-code. Then from proposition 1 , we have

$$
P_{T} \geq \max _{e \in E_{T}} \frac{\left|S^{\prime}\right|}{\left|M \backslash\left\{m_{i}\right\}\right|}=\frac{(c-1)|S|}{|M|-|S|}
$$

Corollary 16. Under assumption 10 and 12,

$$
P_{T} \geq \max \{(c-1) /(l c-1), 1 / h\} . \quad \text { Further, if } c=l=q, \text { then } \quad P_{T} \geq \frac{1}{q+1}
$$

Next, we consider a separable case.

Assumption 17. For $\forall s, M_{s}$ can be grouped into $A_{1}^{s}, A_{2}^{s}, \ldots$ in such a way that $\left|A_{i}^{s} \cap M(f, s)\right|=1$ for $\forall s, \forall A_{i}^{s}$ and $\forall f \in E_{R}$. An $A^{2}$-code given by [14] satisfies this assumption. 
Lemma 18. Under assumption 10, 12 and $17,\left|A_{i}^{s}\right|=l$ and $\left|\left\{A_{i}^{s}\right\}\right|=c$ for $\forall s$.

Proof. The proof is almost the same as that of Theorem 13. We consider a subcode of $\left(\hat{S}, E_{R}, M\right)$ such that the set of messages is restricted to $A_{i}$. In this restricted $A$-code, the number of messages is $\left|A_{i}\right|$ and that of source states is 1 from assumption 17. From Theorem 11, assumption 12 and Theorem 11, even for this restricted $A$-code, $\operatorname{Pr}[\mathrm{R}$ accepts $m]=1 / l$ for $\forall m \in A_{i}$. Then, from Theorem $9,\left|A_{i}^{s}\right|=1 \times l=l$. From Theorem $13,\left|\left\{A_{i}^{s}\right\}\right|=\left|M_{s}\right| /\left|A_{i}\right|=c$

Theorem 19. Under assumption 10, 12 and $17, P_{T} \geq \max \{1 / l, 1 / h\}$.

Proof. It is clear that $P_{T} \geq 1 / h$.

Suppose that the transmitter has $e \in E_{T}$. Let $m_{i}=c\left(s_{i}\right)$ for $i=1,2, \ldots,|S|$. For simplicity, suppose that $m_{i} \in A_{1}^{s_{i}}$ for $i=1,2, \ldots,|S|$. As in the proof of Theorem 15, we consider an $A$-code without splitting $\left(S^{\prime}, E_{R}(e), M \backslash\left\{A_{i}^{s_{i}}\right\}\right)$ such that $\left|S^{\prime}\right|=(c-1)|S|$. Then, as in that proof, we have

$$
P_{T} \geq \max _{e \in E_{T}} \frac{\left|S^{\prime}\right|}{\left|M \backslash\left\{A_{i}^{a_{i}}\right\}\right|}
$$

From assumption 12, and lemma 18,

$$
\left|M \backslash\left\{A_{i}^{s_{i}}\right\}\right|=|M|-\sum\left|A_{1}^{s_{i}}\right|=|M|-l|S|=l c|S|-l|S|
$$

Then, $P_{T} \geq(c-1)|S| /(l c|S|-l|S|)=1 / l$

\subsection{Lower bound on $P_{R_{0}}$ and $P_{R_{1}}$}

Let $E_{T}(f) \triangleq\{e \mid \operatorname{Pr}[\mathrm{T}$ has $e \mid \mathrm{R}$ has $f]>0\}$. Suppose that R has $f$. Then, R knows that $\mathrm{T}$ has some $e \in E_{T}(f)$. Consider an $A$-code $\left(S, E_{T}(f), M(f)\right)$. It is an $A$-code without splitting and without secrecy because the original $A^{2}$-code is so. Let $P_{I}(f)$ and $P_{S}(f)$ denote the impersonation attack probability and the substitution attack probability, respectively. Then it is easy to see that

$$
P_{R_{0}}=\max _{f \in E_{R}} P_{I}(f) \quad P_{R_{1}}=\max _{f \in E_{R}} P_{S}(f)
$$

(Remember that the arbiter accepts $m$ as authentic iff $e$ can generate $m$.) From assumption $10,|M(f)|=c|S|$. Now, from proposition 1, we have Theorem 20.

Theorem 20. Under assumption 10,

1. $P_{R_{0}} \geq 1 / c$

2. If $P_{R_{0}}=1 / c$, then $P_{R_{1}} \geq 1 / c$. 


\subsection{Tightness}

Corollary 21. Suppose that $c=l=q$. Then

1. $P_{I} \geq 1 / q, P_{T} \geq 1 /(q+1), P_{R_{0}} \geq 1 / q$.

2. If $P_{I}=1 / q$, then $P_{S} \geq 1 / q$, If $P_{R_{0}}=1 / q$, then $P_{R_{1}} \geq 1 / q$.

3. Under assumption 17 , if $P_{I}=1 / q$, then $P_{T} \geq 1 / q$.

Corollary 21 is tight because all the bounds are satisfied with equality by the $A^{2}$-codes given by Johansson $[13,14]$.

\subsection{Lower bound on $\left|E_{R}\right|,\left|E_{R} \circ E_{T}\right|$ and $\left|E_{T}\right|$}

In this subsection, we show more tight lower bounds on $\left|E_{R}\right|,\left|E_{R} \circ E_{T}\right|$ and $\left|E_{T}\right|$ than proposition 7 .

Assumption 22. $P_{I}=P_{S}=P_{T}=c|S| /|M|=1 / l$

Theorem 23. Under assumption 10,17 and 22, $\left|E_{R}\right| \geq c|S|(l-1)+1$. The equality holds if and only if the incidence matrix of $E_{R}$ is an orthogonal array $O A(l, c|S|, \lambda)$ where $\lambda=(c|S|(l-1)+1) / l^{2}$ and each $f \in E_{R}$ is used with equal probability.

Proof. From assumption 17, we can consider that our $A$-code without splitting $\left(\hat{S}, E_{R}, M\right)$ is without secrecy. Remember that $|\hat{S}|=c|S|$. For this $A$-code without secrecy, let $\hat{P}_{I}$ and $\hat{P}_{S}$ be the impersonation attack probability and the substitution attack probability, respectively. Then, clearly $\hat{P}_{I}=P_{I}=c|S| /|M|$. From assumption $22, \hat{P}_{S}=\max \left\{P_{S}, P_{T}\right\}=c|S| /|M|$. Now, from proposition 4, we have this Theorem.

Remark. From proposition 7 , we have another bound such that $\left|E_{R}\right| \geq l^{3}$. If $c|S| \geq l^{2}+l+1$, Theorem 23 is more tight than this bound.

Assumption 24. $P_{R_{0}}=P_{R_{1}}=1 / c$

Theorem 25. Under assumption 10 and $24, E_{T}(f) \geq \max \left\{c^{2},|S|(c-1)+1\right\}$.

Proof. From proposition 4.

Theorem 26. Under assumption 10 and 24,

$$
\left|E_{R} \circ E_{T}\right| \geq\left|E_{R}\right| \times \max \left\{c^{2},|S|(c-1)+1\right\} .
$$

Proof. From Theorem 25.

From Theorem 19, if $P_{T}=1 / l$, then $l \leq h=\min _{e}\left|E_{R}(e)\right|$

Assumption 27. For $\forall e \in E_{T},\left|E_{R}(e)\right|=l$

Theorem 28. Under assumption 10, 24 and 27,

$$
\left|E_{T}\right| \geq \frac{\left|E_{R}\right|}{l} \times \max \left\{c^{2},|S|(c-1)+1\right\} .
$$

Proof. From assumption $27, l \times\left|E_{T}\right|=\left|E_{R} \circ E_{T}\right|$. Then, from Theorem 26, Theorem 28 holds. 


\section{Combinatorial bounds for general $A^{2}$-codes}

In this section, we show combinatorial bounds for general $A^{2}$-codes without splitting.

\section{Theorem 29.}

$$
P_{I} \geq \min _{f \in E_{R}} \frac{|M(f)|}{|M|} \quad P_{S} \geq \min _{f \in E_{R}} \frac{|M(f)|-\max _{s \in S}|M(f, s)|}{|M|-\min _{s \in S}|M(f, s)|}
$$

Proof. We consider a splitting $A$-code $\left(S, E_{R}, M\right)$ which corresponds to the original $A^{2}$-code $\left(S, M, E_{R}, E_{T}\right)$ in a natural way (where $|M(f, s)|>1$ ). From proposition 5 , we have this theorem.

Theorem 30. $\quad P_{T} \geq \min _{f \in E_{R}} \frac{|M(f)|-|S|}{|M|-|S|}$

The proof is almost the same as that of theorem 15 .

Theorem 31.

$$
P_{R_{0}} \geq \max _{f \in E_{R}} \frac{|S|}{|M(f)|}
$$

Proof. We consider an $A$-code without splitting $\left(S, E_{T}(f), M(f)\right)$ as shown in subsection 3.3. From proposition 1 , we have this theorem.

Theorem 32.

$$
P_{R_{1}} \geq \max _{f \in E_{R}} \frac{|S|-1}{|M(f)|-\min _{s \in S}|M(f, s)|}
$$

Proof. Fix $f \in E_{R}$ and $m \in M(f)$ arbitrarily. For $\forall m^{\prime} \in M(f)-M(f, f(m))$, let

$$
P_{m^{\prime}} \triangleq \operatorname{Pr}\left[\text { Arbiter accepts } m^{\prime} \mid \mathrm{R} \text { has } f \text { and } \mathrm{T} \text { sends } m\right]
$$

Then

$$
P_{m^{\prime}}=\frac{\sum_{\left\{e \mid m, m^{\prime} \in M(e)\right\}} \operatorname{Pr}\left[E_{T}=e \mid E_{R}=f\right] \operatorname{Pr}[S=f(m)]}{\sum_{\{e \mid m \in M(e)\}} \operatorname{Pr}\left[E_{T}=e \mid E_{R}=f\right] \operatorname{Pr}[S=f(m)]}
$$

Now, we have

$$
\begin{aligned}
& \sum_{m^{\prime} \in M(f)} P_{m^{\prime}} \\
= & \frac{\sum_{m^{\prime} \in M(f)} \sum_{\left\{e \mid \exists s: m^{\prime}=e(s), m \in M(e)\right\}} \operatorname{Pr}\left[E_{T}=e \mid E_{R}=f\right] \operatorname{Pr}[S=f(m)]}{\sum_{\{e \mid m \in M(e)\}} \operatorname{Pr}\left[E_{T}=e \mid E_{R}=f\right] \operatorname{Pr}[S=f(m)]} \\
= & \frac{\sum_{s} \sum_{m^{\prime} \in M(f)} \sum_{\left\{e \mid m^{\prime}=e(s), m \in M(e)\right\}} \operatorname{Pr}\left[E_{T}=e \mid E_{R}=f\right] \operatorname{Pr}[S=f(m)]}{\sum_{\{e \mid m \in M(e)\}} \operatorname{Pr}\left[E_{T}=e \mid E_{R}=f\right] \operatorname{Pr}[S=f(m)]} \\
= & \frac{\sum_{s} \sum_{\{e \mid m \in M(e)\}}\left|\left\{m^{\prime} \mid m^{\prime}=e(s)\right\}\right| \operatorname{Pr}\left[E_{T}=e \mid E_{R}=f\right] \operatorname{Pr}[S=f(m)]}{\sum_{\{e \mid m \in M(e)\}} \operatorname{Pr}\left[E_{T}=e \mid E_{R}=f\right] \operatorname{Pr}[S=f(m)]} \\
= & \frac{\sum_{s} \sum_{\{e \mid m \in M(e)\}} \operatorname{Pr}\left[E_{T}=e \mid E_{R}=f\right] \operatorname{Pr}[S=f(m)]}{\sum_{\{e \mid m \in M(e)\}} \operatorname{Pr}\left[E_{T}=e \mid E_{R}=f\right] \operatorname{Pr}[S=f(m)]} \\
= & |S|
\end{aligned}
$$


Further,

$$
\begin{aligned}
& \sum_{m^{\prime} \in M(f, f(m))} P_{m^{\prime}} \\
= & \frac{\sum_{m^{\prime} \in M(f, f(m))} \sum_{\left\{e \mid \exists s: m^{\prime}=e(s), m \in M(e)\right\}} \operatorname{Pr}\left[E_{T}^{\prime}=e \mid E_{R}=f\right] \operatorname{Pr}[S=f(m)]}{\sum_{\{e \mid m \in M(e)\}} \operatorname{Pr}\left[E_{T}=e \mid E_{R}=f\right] \operatorname{Pr}[S=f(m)]} \\
= & \frac{\sum_{m^{\prime} \in M(f, f(m))} \sum_{\left\{e \mid m^{\prime}=e(f(m)), m \in M(e)\right\}} \operatorname{Pr}\left[E_{T}=e \mid E_{R}=f\right] \operatorname{Pr}[S=f(m)]}{\sum_{\{e \mid m \in M(e)\}} \operatorname{Pr}\left[E_{T}=e \mid E_{R}=f\right] \operatorname{Pr}[S=f(m)]} \\
= & \frac{\sum_{\{e \mid m \in M(e)\}}\left|\left\{m^{\prime} \mid m^{\prime}=e(f(m))\right\}\right| \operatorname{Pr}\left[E_{T}=e \mid E_{R}=f\right] \operatorname{Pr}[S=f(m)]}{\sum_{\{e \mid m \in M(e)\}} \operatorname{Pr}\left[E_{T}=e \mid E_{R}=f\right] \operatorname{Pr}[S=f(m)]} \\
= & \frac{\sum_{\{e \mid m \in M(e)\}} \operatorname{Pr}\left[E_{T}=e \mid E_{R}=f\right] \operatorname{Pr}[S=f(m)]}{\sum_{\{e \mid m \in M(e)\}} \operatorname{Pr}\left[E_{T}=e \mid E_{R}=f\right] \operatorname{Pr}[S=f(m)]} \\
= & 1
\end{aligned}
$$

Hence, $\quad \sum_{m^{\prime} \in M(f) \backslash M(f, f(m))} P_{m^{\prime}}=\sum_{m^{\prime} \in M(f)} P_{m^{\prime}}-\sum_{m^{\prime} \in M(f, f(m))} P_{m^{\prime}}=|S|-1$

Therefore, there exists $\hat{m}^{\prime} \in M(f) \backslash M(f, f(m))$ such that

$$
P_{\dot{m}^{\prime}} \geq \frac{|S|-1}{|M(f)|-|M(f, f(m))|}
$$

Thus

$$
\begin{aligned}
P_{R_{1}} & \triangleq \max _{f \in E_{R}^{\prime}} \sum_{m \in M} \operatorname{Pr}(m) \max _{\hat{m} \neq m} \operatorname{Pr}[\text { Arbiter accepts } \hat{m} \mid \mathrm{R} \text { has } f \text { and } \mathrm{T} \text { sends } m] \\
& \geq \max _{f \in E_{R}} P_{\dot{m}^{\prime}} \\
& \geq \max _{f \in E_{R}} \frac{|S|-1}{|M(f)|-\min _{s \in S}|M(f, s)|}
\end{aligned}
$$

\section{Another definition of $\boldsymbol{P}_{R_{\mathrm{o}}}$ and $\boldsymbol{P}_{R_{1}}$}

We can define $P_{R_{0}}$ and $P_{R_{1}}$ in a different way from eq.(2.4) and eq.(2.5). The alternative definitions are

$$
\begin{aligned}
P_{R_{0}} \triangleq & \sum_{f \in E_{R}} \operatorname{Pr}(f) \max _{m \in M} \operatorname{Pr}\left[\text { Arbiter (or T) accepts } m \mid \mathrm{R} \text { has } f \in E_{R}\right] \\
P_{R_{1}} \triangleq & \sum_{f \in E_{R}} \operatorname{Pr}(f) \sum_{m \in M} \operatorname{Pr}(m) \\
& \max _{\hat{m} \neq m} \operatorname{Pr}[\text { Arbiter (or T) accopts } \hat{m} \mid \mathrm{R} \text { has } f \text { and } \mathrm{T} \text { sends } m]
\end{aligned}
$$

For the model without secrecy, the lower bounds on the above $P_{R_{0}}$ and $P_{R_{1}}$ are the same as those on the original $P_{R_{0}}$ and $P_{R_{1}}$. For the gencral model, we obtain the following bound. 
Theorem 33.

$$
P_{R_{0}} \geq \min _{f \in E_{R}} \frac{|S|}{|M(f)|} \quad P_{R_{1}} \geq \min _{f \in E_{R}} \frac{|S|-1}{|M(f)|-\min _{s} \in S|M(f, s)|}
$$

\section{References}

1. G.J.Simmons, "A survey of Information Authentication", in Contemponary Cryptology, The science of information integrity, ed. G.J.Simmons, IEEE Press, New York, 1992.

2. G.J.Simmons, "Authentication theory/coding theory", Proceedings of Crypto'84, Lecture Notes in Computer Science, LNCS 196, Springer Verlag, pp.411-431 (1985).

3. E.F.Brickell, "A few results in message authentication", Congresus Numerantium, vol.43, pp.141-154 (1984).

4. G.J.Simmons, "Message authentication: a game on hypergraphs", Congresus Numerantium, vol.45, pp.161-192 (1984).

5. J.L.Massey, Cryptography - a selective survey, in Digital Communications, North Holland (pub.), pp.3-21, (1986).

6. D.R.Stinson, "Some constructions and bounds for authentication codes", Journal of Cryptology, Vol.1, 1988, pp.37-51, (1988).

7. D.R.Stinson, "The combinatorics of authentication and secrecy codes", Journal of Cryptology, Vol.2, no 1, 1990, pp.23-49, (1990).

8. D.R.Stinson, "Combinatorial Characterization of Authentication Codes", Proceedings of Crypto'91, Lecture Notes in Computer Science, LNCS 576, Springer Verlag, pp62-72 (1992).

9. Marijke De Soete, "New Bounds and Constructions for Authentication/Secrecy Codes with Splitting”, Journal of Cryptology, Vol.3, no 3, 1991, pp173-186 (1991).

10. G.J.Simmons, "Message Authentication with Arbitration of Transmitter/Receiver Disputes", Proceedings of Eurocrypt'87, Lecture Notes in Computer Science, LNCS 304, Springer Verlag, pp.150-16 (1987)

11. G.J.Simmons, "A Cartesian Product Construction for Unconditionally Secure Authentication Codes that Permit Arbitration", Journal of Cryptology, Vol.2, no.2, 1990, pp.77-104 (1990).

12. Thomas Johansson, "Lower Bounds on the Probability of Deception in Authentication with Arbitration", In Proceedings of 1993 IEEE International Symposium on Information Theory, San Antonio, USA, January 17-22, 1993, pp.231.

13. Thomas Johansson, "Lower Bounds on the Probability of Deception in Authentication with Arbitration", submitted to IEEE Trans. on IT (private communication).

14. Thomas Johansson, "On the construction of perfect authentication codes that permit arbitration", Proceedings of Crypto'93, Lecture Notes in Computer Science, LNCS 773, Springer Verlag, pp.341-354 (1993).

15. K.Kurosawa, "New bound on authentication code with arbitration", Proceedings of Crypto'94, Lecture Notes in Computer Science, LNCS 899, Springer Verlag, pp.140-149 (1994). 